\title{
Prognostic Factors and Clinical Outcome in Parotid Gland Tumors: a Single Institution Experience from the Eastern Black Sea Region of Turkey
}

\author{
Mustafa Kandaz*, Gulsen Soydemir, Zumrut Bahat, Emine Canyılmaz, Adnan \\ Yoney
}

\begin{abstract}
Purpose: To review clinical characteristics, treatment outcomes and prognostic factors in patients with parotid gland tumors treated with surgery and postoperative radiotherapy. Materials and Methods: We retrospectively reviewed 69 patients with parotid gland tumors, with a median follow-up of 52 months (range, 2-228 months). and a median radiotherapy dose of $60 \mathrm{~Gy}$ (range, $30-69 \mathrm{~Gy})$. Results: There were $24(35 \%)$ females and $45(65 \%)$ males, at a ratio of 1/1.9. Median age at presentation was 58.9 \pm 17.2 (range 13-88) years. The most common histology was adenoid cystic carcinoma (33\%) and mucoepidermoid carcinoma (28\%). The mean overall survival (OS) was $65.3 \pm 8(95 \%$ confidence interval $[\mathrm{CI}], 49.6-81.1)$ months and the median overall survival was $40.0 \pm 7$ (95\% CI, 26.2-53.7) months. The $-1,-3,-5$ and -10 year $O S$ rates were $78 \%, 52.4 \%, 35.3 \%$ and $19.6 \%$ respectively. The mean disease free survival (DFS) was 79.2 \pm 10 (95\% CI, 59.3-97.1) months and the median disease free survival was 38 $\pm 13(95 \%$ CI, 7.05-88.7) months. The $-1,-3,-5$ and -10 year DFS rates were $71.9 \%, 50.1 \%, 43.7 \%$ and $30.1 \%$ respectively. On univariate analysis, the $O S$ was significantly better with female $\operatorname{sex}(p<0.005),<50$ age $(p<0.021)$, T stage $(\mathbf{p}<0.0001)$, absence of lymph node involvement $(\mathbf{p}<0.0001)$, lower tumor grade $(\mathbf{p}<0.0001)$, absence of lymphovascular invasion $(\mathbf{p}<0.002)$, absence of perineural invasion $(p<0.0001)$, absence of extracapsuler extension $(p<0.0001)$, surgical margin negativity $(p<0.006), \leq 60$ Gy radiotherapy dose $(p<0.0001)$ and absence of distant metastasis $(p<0.027)$. Conclusions: Employing existing standards of postoperative radiotherapy is a possible treatment that was found to be mainly effective in patients with parotid gland carcinomas.
\end{abstract}

Keywords: Parotid gland cancers - patients characteristics - clinical outcome - prognostic factors

Asian Pac J Cancer Prev, 17 (3), 1169-1174

\section{Introduction}

Parotid carcinoma is very heterogeneous and rare disease. Approximately $4 \%$ of head-and neck-cancers and $0.5 \%$ of all human cancers (Al-Mamgani et al., 2012). Mukoepidermoid carcinoma, squamous cell carcinoma, adenocarcinoma and adenoid cystic carcinomas are the most histological types (Alterio et al., 2011).

The mainstay treatment is surgical resection and in well-selected patients with low grade tumor removed with resection free margins $\geq 5 \mathrm{~mm}$, early-stage parotidectomy alone might be sufficient. Other patients, combined modality treatment of surgery and postoperative radiotherapy (RT) is needed (1). Some different studies shown that, surgery and postoperative RT (PORT) improved locoregional control (LRC), overall survival (OS) and disease-free survival (DFS) compared to one modality alone (Al-Mamgani et al., 2012).
PORT is indicated for patients with tumor size $>4 \mathrm{~cm}$, tumor with deep lobe settlement, high grade tumor, lymph node involvement, advanced stage, positive surgery margins, perivascular and perineural invasion, nerve infiltration, recurrent tumor and sof tissue extension (Alterio et al., 2011).

In this study was evaluate clinical outcomes and prognostic factors in patietns with parotid carcinomas treated with surgery and RT at our institution.

\section{Materials and Methods}

\section{Patients selection}

We retrospectivly analyzed the data of 69 patiens treated with surgery and RT for parotid carcinomas from January 1997 and December 2014. Locoregional clinical staging was performed using clinical examinations and computed tomography (CT) and/or magnetic resonance 
(MR) and/or PET-CT. Distant metastases were detected using total-body CT, abdominal ultrasonography (USG) and/or MR. Surgical procedure (superficial or total parotidectomy, lymph node dissection) were recorded. Different surgical approaches depended on tumor location, histological characteristic and stage. Ipsilateral neck disection was performed in patients of high-grade tumors, radiological or clinical evidence of lymph node metastasis or with the risk of lymph node involvement.

\section{Treatment}

PORT was given to patients in advanced stage, positive surgical margins, recurrent tumor, high grade histology and presence of lymph node metastasis. Ipsilateral neck lymph nodes were irradiated in patients of pathological lymph nodes or, as elective treatment, in high-risk patients. Total PORT dose was planned ranged from 60 Gy to 66 Gy for negative and positive microscopic surgical bed margins, respectively, and from 50 Gy to 60 Gy for negative and positive nodes with or without extracapsular extension, respectively. RT was performed with linear accelerator of $6 \mathrm{MV}$ - energy and/or cobalt-60. RT was performed with some patients IMRT and some cases with 2-D and/or 3-D confomal technique. One daily fraction of 1.8 to 2 Gy for 5 days/week was used for all patients. When the tumor infiltrated the skin or was located in the superficial lobe, a waterequivalent bolus was added for all of the treatment duration and/or a mixed photon-electron beam technique was used according to personalized treatment plans. The spinal cord doses was limited between 45 Gy to 50 Gy. Head and neck mask was used for immobilization, simulation of all therapy phases. When computed tomography-based treatment was planned, organ at risk contouring and dose prescription and target volume were performing according to the International Commission on Radiation Units (ICRU) 62 guidelines. Radiotherapy-related acute and late mucosa, soft tissue, skin and temporomandibular joint toxicity was commentate according to the Radiation Therapy Oncology Group/European Organization for Research and Treatment of Cancer (RTOG/EORTC) scoring system.

\section{Follow-up}

Follow-up was performed every 3 months for the first 2 years, every 6 months for the subsequent 3 years, and once a year thereafter. Clinical and radiological examinations were performed for each visit.

\section{Statistical methods}

In this study, clinical outcome was evaluated of diease-free and overall survival. Disease-free survival was evaluated between the end of PORT and the occurrence of local and/or distant recurrence. The association between clinical outcome (distant metastasis and local recurrence) and tumor characteristics (stage, grade, lynph node metastasis, positive surgical margins, extracapsular extension and perineural invasion) were analyzed. The association between total dose applied, surgical technique and toxicity (mucosa, soft tissue and skin) was also appraised.

Survival rates were calculated using Kaplan-Meier technique. All significant tests and statistical significance were accepted for a calculated $\mathrm{p}$ value of $<0.005$.

\section{Results}

\section{Patients characteristics}

A total of 69 patients of parotid carsinomas. There were $24(35 \%)$ females and $45(65 \%)$ males, for a ratio of $1 / 1.9$. Median age at presentation was $58.9 \pm 17.2$ (range 13 to 88 ) years. Of the 69 patients, 18 (26\%) occurred in $<50$ years and $51(74 \%)$ in $\geq 50$ years. The most common histology was adenoid cystic carcinoma $(33 \%)$, mucoepidermoid carcinoma $(28 \%)$, malign mixt tumor $(18.5 \%)$ and squamous cell carcinoma (10\%). The rarer histology was undifferentiated carcinom $(3 \%)$, ductal carcinom (1.5\%), pleomorphic carcinom (1.5\%), acinic cell carcinom $(1.5 \%)$, adenosquamous carcinom $(1.5 \%)$ and papiller adenocarcinom (1.5\%). Pathological T stage: $2(3 \%)$ patients in stage I, $32(46 \%)$ patients in stage II, $29(42 \%)$ patients in stage III and $6(9 \%)$ patients in stage IV.39 (57\%) patients had lymph node involvement. Pathological grade were low grade in $5(7 \%)$ patients, intermediate grade in $42(61 \%)$ patients and high grade in $22(32 \%)$ patients. Lymphovasculer invasion was seen in $29(42 \%)$ patients. Perineural invasion was seen in $33(48 \%)$ patients. $4(6 \%)$ of patients had extracapsuler spread.

\section{Treatment characteristics}

Gland excision was performed to all patients. 8 (12\%) patients underwent unilateral functional neck dissection, $27(39 \%)$ patients underwent unilateral radical neck dissection. Surgical margins were negative in $30(43 \%)$, positive in $31(45 \%)$ and close $(<5 \mathrm{~mm})$ in $8(12 \%)$ patients.

All patients (one patient with benign parotid tumors, radiotherapy was indicated only in case of recurrent disease) were treated with surgery followed by

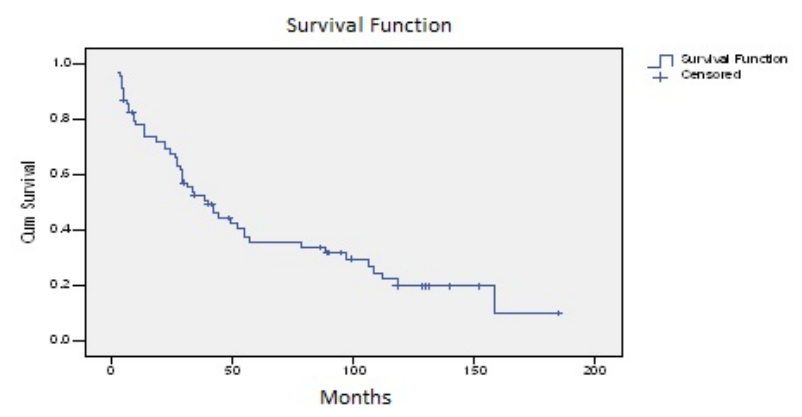

Figure 1. Overall Survival

\section{Table 1. Acute Toxicity}

\begin{tabular}{lccc}
\hline & & Skin lesions & Oral mucositis \\
\hline Grade 0-patients & $(\%)$ & 0 & 0 \\
Grade I-patients & $(\%)$ & $12(17.4 \%)$ & $16(23.2 \%)$ \\
Grade II-patients & $(\%)$ & $38(55.1 \%)$ & $39(56.5 \%)$ \\
Grade III-patients & $(\%)$ & $13(18.8 \%)$ & $10(14.5 \%)$ \\
Grade IV-patients & $(\%)$ & $6(8.7 \%)$ & $4(5.8 \%)$ \\
\hline
\end{tabular}


Table 2. Patient Characteristics of Log-rank Univariate Analysis for Overall Survival

\begin{tabular}{|c|c|c|c|c|c|c|c|}
\hline & $\begin{array}{c}\text { Mean } \\
\text { (month) } \\
95 \% \mathrm{CI}\end{array}$ & $\begin{array}{c}\text { Median } \\
\text { (month) } \\
95 \% \mathrm{CI}\end{array}$ & $\mathrm{p}$ & $\begin{array}{c}\text { Survival rate } \\
1 \text { year }(\%)\end{array}$ & $\begin{array}{c}\text { Survival rate } \\
3 \text { year }(\%)\end{array}$ & $\begin{array}{c}\text { Survival rate } \\
5 \text { year }(\%)\end{array}$ & $\begin{array}{c}\text { Survival rate } \\
10 \text { year }(\%)\end{array}$ \\
\hline General & $\begin{array}{c}65.3 \pm 8 \\
49.6-81.1\end{array}$ & $\begin{array}{c}40.0 \pm 7 \\
26.2-53.7\end{array}$ & & 78 & 52.4 & 35.3 & 19.6 \\
\hline Gender & & & & & & & \\
\hline Female & $\begin{array}{c}100.0 \pm 15 \\
70.6-130.7\end{array}$ & $\begin{array}{c}97.0 \pm 23 \\
51.3-142.6\end{array}$ & \multirow{2}{*}{0.005} & 91.7 & 64.9 & 60.3 & 35.3 \\
\hline Male & $\begin{array}{c}46.6 \pm 7 \\
31.3-61.9\end{array}$ & $\begin{array}{c}29.0 \pm 6 \\
16.1-41.8\end{array}$ & & 70.8 & 45.6 & 20.8 & 10.7 \\
\hline \multicolumn{8}{|l|}{ Age } \\
\hline$<50$ & $\begin{array}{c}84.4 \pm 11 \\
61.4-107.5\end{array}$ & $\begin{array}{c}106.0 \pm 41 \\
24.2-187.7\end{array}$ & \multirow{2}{*}{0.021} & 88.9 & 82.5 & 50.8 & 30.5 \\
\hline$\geq 50$ & $\begin{array}{c}55.0 \pm 8 \\
37.8-72.2\end{array}$ & $\begin{array}{c}29.0 \pm 2 \\
24.1-33.8\end{array}$ & & 72.1 & 39.1 & 30 & 15.6 \\
\hline \multicolumn{8}{|l|}{ Histology } \\
\hline Adenoid cystic $\mathrm{Ca}$ & $\begin{array}{c}58.5 \pm 9 \\
40.7-76.4\end{array}$ & $\begin{array}{c}52.0 \pm 11 \\
30.0-73.9\end{array}$ & \multirow{10}{*}{0.524} & 78.3 & 60.9 & 37.7 & 8.8 \\
\hline Mucoepidermoid Ca & $\begin{array}{c}84.7 \pm 18 \\
49.0-120.4\end{array}$ & $\begin{array}{c}44.0 \pm 35 \\
0.0-114.4\end{array}$ & & 89.5 & 53.7 & 46 & 30.7 \\
\hline Malign mixt tumor & $\begin{array}{c}66.1 \pm 19 \\
28.1-104.0\end{array}$ & $\begin{array}{l}34.0 \pm 19 \\
0.0-72.5\end{array}$ & & 69.2 & 46.2 & 36.9 & 27.7 \\
\hline Squamous cell $\mathrm{Ca}$ & $\begin{array}{l}30.8 \pm 11 \\
8.9-52.7\end{array}$ & $\begin{array}{c}18.0 \pm 6 \\
5.1-30.8\end{array}$ & & 71.4 & 42.9 & 14.3 & - \\
\hline Ductal Ca & - & - & & - & - & - & - \\
\hline Undifferentiated $\mathrm{Ca}$ & - & - & & 50 & - & - & - \\
\hline Pleomorphic $\mathrm{Ca}$ & - & - & & - & - & - & - \\
\hline Acinic cell Ca & - & - & & - & - & - & - \\
\hline Adenosquamous $\mathrm{Ca}$ & - & - & & - & - & - & - \\
\hline $\begin{array}{l}\text { Papiller Adeno } \mathrm{Ca} \\
\mathrm{T} \text { stage }\end{array}$ & \multicolumn{4}{|c|}{ T stage } & & - & - \\
\hline I & $\begin{array}{c}68.0 \pm 44 \\
0.0-155.3\end{array}$ & $\begin{array}{l}5 \\
-\end{array}$ & \multirow{4}{*}{0.0001} & 50 & 50 & 50 & 50 \\
\hline II & $\begin{array}{c}103.9 \pm 12 \\
78.7-129.1\end{array}$ & $\begin{array}{c}108.0 \pm 14 \\
79.0-136.9\end{array}$ & & 90.4 & 76.7 & 65.5 & 40.7 \\
\hline III & $\begin{array}{c}32.2 \pm 5 \\
20.8-43.7\end{array}$ & $\begin{array}{c}27.0 \pm 3 \\
19.9-34.0\end{array}$ & & 72.4 & 31 & 9.1 & - \\
\hline IV & $\begin{array}{c}20.8 \pm 9 \\
2.4-39.2\end{array}$ & $\begin{array}{c}6.0 \pm 4 \\
0.0-15.6\end{array}$ & & 50 & 33.3 & - & - \\
\hline \multicolumn{8}{|l|}{ N stage } \\
\hline Negative & $\begin{array}{c}90.5 \pm 12 \\
67.0-114.1\end{array}$ & $\begin{array}{c}78.0 \pm 31 \\
16.9-139.0\end{array}$ & \multirow{2}{*}{0.0001} & 84.4 & 65.7 & 53.1 & 35.2 \\
\hline Positive & $\begin{array}{c}34.6 \pm 6 \\
21.8-47.3\end{array}$ & $\begin{array}{c}26.0 \pm 3 \\
18.6-33.3\end{array}$ & & 69.7 & 34.8 & 13.9 & 3.5 \\
\hline \multicolumn{8}{|l|}{ Grade } \\
\hline Low & $\begin{array}{r}165.7 \pm 16 \\
133-198,4\end{array}$ & - & & - & - & - & 75 \\
\hline Intermediate & $\begin{array}{c}67.5 \pm 8 \\
50.0-85.0\end{array}$ & $\begin{array}{c}44.0 \pm 7 \\
30.0-57.9\end{array}$ & 0.0001 & 88 & 58.5 & 37.3 & 20.2 \\
\hline High & $\begin{array}{c}31.3 \pm 8 \\
14.9-47.8\end{array}$ & $\begin{array}{c}13.0 \pm 2 \\
7.1-18.8\end{array}$ & & 53.6 & 29.2 & 16.2 & - \\
\hline \multicolumn{8}{|c|}{ Lymphovasculer invasion } \\
\hline Yes & $\begin{array}{c}36.7 \pm 7 \\
22.1-51.4\end{array}$ & $\begin{array}{c}27.0 \pm 4 \\
18.2-35.7\end{array}$ & \multirow{2}{*}{0.002} & 65.5 & 31 & 15.8 & 7.9 \\
\hline No & $\begin{array}{c}85.2 \pm 11 \\
62.9-107.5\end{array}$ & $\begin{array}{c}78.0 \pm 25 \\
27.2-128.7\end{array}$ & & 87.4 & 68.6 & 50.4 & 29.6 \\
\hline \multicolumn{8}{|l|}{ Perineural invasion } \\
\hline Yes & $\begin{array}{c}30.3 \pm 5 \\
18.6-42.0\end{array}$ & $\begin{array}{c}26.0 \pm 7 \\
10.6-41.3\end{array}$ & \multirow{2}{*}{0.0001} & 63.2 & 22.1 & 11.9 & - \\
\hline No & $\begin{array}{c}96.9 \pm 11 \\
73.6-120.1\end{array}$ & $\begin{array}{c}97.0 \pm 29 \\
39.3-154.6\end{array}$ & & 91.6 & 80 & 57 & 36.4 \\
\hline \multicolumn{8}{|c|}{ Extracapsuler extansion } \\
\hline Yes & $\begin{array}{c}10.2 \pm 5 \\
0.0-21.3\end{array}$ & $\begin{array}{c}4.0 \pm 2 \\
0.0-7.9\end{array}$ & \multirow{2}{*}{0.0001} & 25 & - & - & - \\
\hline No & $\begin{array}{c}68.8 \pm 8 \\
52.4-85.2 \\
\end{array}$ & $\begin{array}{c}42.0 \pm 9 \\
24.0-59.9 \\
\end{array}$ & & 81.3 & 55.7 & 37.5 & 20.8 \\
\hline
\end{tabular}


Table 3. Treatment Results of Log-rank Univariate Analysis for Overall Survival

\begin{tabular}{|c|c|c|c|c|c|c|c|}
\hline & $\begin{array}{c}\text { Mean } \\
\text { (month) }\end{array}$ & $\begin{array}{l}\text { Median } \\
\text { (month) }\end{array}$ & \multirow[t]{2}{*}{$\mathrm{p}$} & \multirow{2}{*}{$\begin{array}{c}\text { Survival rate } \\
1 \text { year }(\%)\end{array}$} & \multirow{2}{*}{$\begin{array}{l}\text { Survival rate } \\
3 \text { year }(\%)\end{array}$} & \multirow{2}{*}{$\begin{array}{l}\text { Survival rate } \\
5 \text { year }(\%)\end{array}$} & \multirow{2}{*}{$\begin{array}{l}\text { Survival rate } \\
10 \text { year }(\%)\end{array}$} \\
\hline & $95 \% \mathrm{CI}$ & $95 \% \mathrm{CI}$ & & & & & \\
\hline \multicolumn{8}{|c|}{ Surgical margin } \\
\hline Negative & $\begin{array}{c}82.9 \pm 12 \\
58.0-107.8\end{array}$ & $\begin{array}{c}52.0 \pm 32 \\
0.0-116.2\end{array}$ & \multirow{3}{*}{0.006} & 86.2 & 68.2 & 45.2 & 28.8 \\
\hline Positive & $\begin{array}{c}38.6 \pm 7 \\
23.9-53.2\end{array}$ & $\begin{array}{c}27.0 \pm 5 \\
17.0-36.9\end{array}$ & & 67.7 & 35.5 & 19.7 & 5.3 \\
\hline Close & $\begin{array}{c}67.9 \pm 14 \\
40.0-95.7\end{array}$ & - & & 87.5 & 60 & 60 & 60 \\
\hline \multicolumn{8}{|c|}{ Radiotherapy dose } \\
\hline$\leq 60 \mathrm{~Gy}$ & $\begin{array}{c}89.5 \pm 11 \\
66.9-112.1\end{array}$ & $\begin{array}{c}97.0 \pm 38 \\
21.8-172.1\end{array}$ & \multirow{2}{*}{0.0001} & 85.4 & 67.6 & 50.5 & 33.9 \\
\hline$>60 \mathrm{~Gy}$ & $\begin{array}{c}30.5 \pm 5 \\
19.4-41.6\end{array}$ & $\begin{array}{c}24.0 \pm 5 \\
12.5-35.4\end{array}$ & & 66.7 & 29.6 & 12.7 & - \\
\hline \multicolumn{8}{|c|}{ Concurrent chemotherapy } \\
\hline Yes & $\begin{array}{c}62.7 \pm 33 \\
0.0-128.1\end{array}$ & $\begin{array}{l}29.0 \pm 25 \\
0.0-78.0\end{array}$ & \multirow{2}{*}{0.988} & 75 & 50 & 25 & 25 \\
\hline No & $\begin{array}{c}67.5 \pm 8 \\
50.4-84.6\end{array}$ & $\begin{array}{c}40.0 \pm 6 \\
26.6-53.3\end{array}$ & & 78 & 52.4 & 36 & 18.9 \\
\hline \multicolumn{8}{|c|}{ Local recurrence } \\
\hline Yes & $\begin{array}{c}67.9 \pm 14 \\
39.7-96.1\end{array}$ & $\begin{array}{c}52 \\
26.3-77.6\end{array}$ & \multirow{2}{*}{0.633} & 88.2 & 58.8 & 30.3 & 22.7 \\
\hline No & $\begin{array}{c}65.5 \pm 9 \\
46.2-84.7\end{array}$ & $\begin{array}{c}38.0 \pm 6 \\
25.0-50.9\end{array}$ & & 70.7 & 50.4 & 36.7 & 18 \\
\hline \multicolumn{8}{|c|}{ Distant metastasis } \\
\hline Yes & $\begin{array}{c}46.2 \pm 8 \\
29.4-63.0\end{array}$ & $\begin{array}{c}31.0 \pm 6 \\
17.9-44.0\end{array}$ & \multirow{2}{*}{0.027} & 67.7 & 45.2 & 21.3 & 8.5 \\
\hline No & $\begin{array}{c}86.6 \pm 12 \\
61.2-111.7\end{array}$ & $\begin{array}{c}49.0 \pm 32 \\
0.0-113.4\end{array}$ & & 86.8 & 58.7 & 48.7 & 30.5 \\
\hline \multicolumn{8}{|c|}{ Treatment options } \\
\hline Primer & $\begin{array}{c}67.7 \pm 9 \\
49.7-85.7\end{array}$ & $\begin{array}{c}40.0 \pm 7 \\
25.6-54.3\end{array}$ & \multirow{2}{*}{0.929} & 75.5 & 53.8 & 37.5 & 18.3 \\
\hline Relaps & $\begin{array}{c}59.9 \pm 18 \\
23.4-96.3\end{array}$ & $\begin{array}{l}33.0 \pm 14 \\
5.1-60.8\end{array}$ & & 81.8 & 45.5 & 22.7 & 22.7 \\
\hline
\end{tabular}

postoperative radiotherapy (PORT). Radiation dose were given $42(61 \%)$ patients $\leq 60$ Gy and $27(39 \%)$ patiens $>60$ Gy. The fractional dose 1.8/2Gy daily. 9 (13\%) patients received tumor bed irradiation. $52(75 \%)$ patients were treated with tumor bed and unilateral neck nodal irradiation and $8(12 \%)$ patients were treated with tumor bed and bilateral neck nodal irradiation. $5(7 \%)$ patients underwent concurrent chemotheraphy.

\section{Toxicity}

Acute toxicity was noted by seeing skin lesions and oral mucositis. $12(17.4 \%)$ patients had grade I skin lesions, $38(55.1 \%)$ patients had grade II, 13 (18.8\%) patients had grade III and $6(8.7 \%)$ patients hade grade IV. $16(23.2 \%)$ patients had grade I oral mucositis, $39(56.5 \%)$ patients had grade II, $10(14.5 \%)$ patients had grade III and $4(5.8 \%)$ patients had grade IV. Acute toxicity was shown in table 1 . None of the patients developed grade 3 and 4 late toxicity of soft tissues, skin or oral mucosa.

\section{Locoregional recurrens and distant metastasis}

Median follow-up 52 months (range, 2-228 months). $17(25 \%)$ patients developed locoregional recurrrens (5126 months). 12 of the relapse cases were seen in treatment area and 5 of them were in the neighbourhood. The locoregional recurrences cases included, 12 (71\%) male, 6 (35\%) adenoid cystic carcinom, 5 (29\%) mucoepidermoid carcinom, 8 (47\%) lymph node involvement, 7 (41\%) positive surgical margin, $6(35 \%)$ lymphovasculer invasion, 7 (41\%) perineural invasion and grade 2-3 was seen in all patients. After locoregional recurrence was average survival 30 (range, 2-96) months.

$31(45 \%)$ patients developed distant metastasis to the lungs in $19(61 \%)$ patients ( lungs plus bone in 2, lungs plus brain 1 , lungs plus liver 3$)$, brain in $4(13 \%)$ patients, liver in $1(3.5 \%)$ patients, bone in $6(19 \%)$ patients and mediastinal lymph node metastasis in $1(3.5 \%)$ patients (9 patients had both locoregional and distant metastasis). The distant metastasis cases included, 22 (71\%) male, $13(42 \%)$ adenoid cystic carcinom, $8(26 \%)$ malign mixt tumor, 18 (58\%) lymph node involvement, 17 (55\%) positive surgical margin, $15(48 \%)$ lymphovasculer invasion, $16(52 \%)$ perineural invasion. After distant metastasis was average survival 18 (range, 0-86) months.

\section{Survival analysis}

The mean overall survival (OS) $65.3 \pm 8$ (95\% confidence interval [CI], 49.6-81.1) months and the median overall survival $40.0 \pm 7(95 \% \mathrm{CI}, 26.2-53.7)$ months. The $-1,-3,-5$ and -10 year OS rates were $78 \%$, 
$52.4 \%, 35.3 \%$ and $19.6 \%$ respectively (figure 1.) (table 2.).

The mean disease free survival (DFS) $79.2 \pm 10(95 \%$ CI, 59.3-97.1) months and the median disease free survival $38 \pm 13$ (95\% CI, 7.05-88.7) months. The $-1,-3,-5$ and -10 year DFS rates were $71.9 \%, 50.1 \%, 43.7 \%$ and $30.1 \%$ respectively.

On univariate analysis, the OS was significantly better with female sex $(\mathrm{p}<0.005),<50$ age $(\mathrm{p}<0.021), \mathrm{T}$ stage $(\mathrm{p}<0.0001)$, absence of lymph node involvement $(\mathrm{p}<0.0001)$, lower tumor grade $(\mathrm{p}<0.0001)$, absence of lymphovasculer invasion ( $\mathrm{p}<0.002)$, absence of perineural invasion $(\mathrm{p}<0.0001)$, absence of extracapsuler extansion $(\mathrm{p}<0.0001)$, surgical margin negativity $(\mathrm{p}<0.006), \leq 60$ Gy radiotherapy dose $(\mathrm{p}<0.0001)$ and absence of distant metastasis $(\mathrm{p}<0.027)$ (Table 3.).

\section{Discussion}

Parotid tumors represent $1-5 \%$ of all head and neck tumors and 0.1-0.5 of all human tumors (Lima et al., 2005; Kaur et al., 2014). Malignant parotid neoplasms have low incidence and heterogeneity of histological types (Mercante et al., 2014). Our retrospective study included 69 patients who had surgery and PORT. We evaluated in this study clinical outcome and the incidence of acute toxicity.

Parotid glands tumors occurs more commonly between the 6. and 7. decades (Alterio et al., 2011; Al-Mamgani et al., 2012; Mercante et al., 2014). The mean age in our study was, 58.9 years. The male/female ratio in our study was $1.9 / 1$.

Various studies have shown mucoepidermoid carcinoma, adenoid cystic carcinoma and adenocarcinoma are the most common histology affecting parotid glands (Nascimento et al., 1986; Feinstein et al., 2011; AlMamgani et al., 2012; Mercante et al., 2014). The most frequently recorded histological types in our series were adenoid cystic carcinoma followed by mucoepidermoid carcinoma and malign mixt tumor.

Parotid gland's cancers is mostly diagnosed at a locally advanced stage (Ito et al., 2005; Al-Mamgani et al., 2012; Mercante et al., 2014). In our study; $46 \%$ occured in stage $\mathrm{T} 2,42 \%$ stage $\mathrm{T} 3$ and $9 \%$ stage T4.

Some studies have shown that the lymphndode is involved at the time of diagnosis (Koivunen et al., 2002), but some studies have shown often there is lymph node involvement at the time of diagnosis (Al-Mamgani et al., 2012; Mercante et al., 2014). In our series, was $57 \%$ had lymph node involvement. Parotid glands cancers oftenly dont have extracapsuler extansion at the time of diagnosis (Mercante et al., 2014). In our study, there was 6\% extracapsuler extansion. Parotid glands cancers is mostly diagnosed at intermediate and high grade (Al-Mamgani et al., 2012). In our series, $61 \%$ occured in intermediate grade and $32 \%$ high grade.

Surgery and/or neck disection remains the primary treatment option for parotid malignancies (Al-Mamgani et al., 2012; Mercante et al., 2014). In our study there was $51 \%$ neck disection in patients.

Radiotherapy doses, of at least $60 \mathrm{~Gy},>65 \mathrm{~Gy}$ and 70 Gy were thus recommended for microscopically negative and positive margins and gross residual disease, respectively (Terhaard et al., 2005). In our study applied the same protocol.

Different acute and late treatment toxicities have been described when RT was delivered to the patients (Garden et al., 1997) soft tissue fibrosis, bone and temporal lobe necrosis, hearing loss, frozen temporomandibular joint, brachial plexopathy. These toxicities could be related to different factors: dose, fraction, radiotherapy technique and size of treatment field. In our study, the acute toxicity was observed in all patients, but late toxicity was not observed.

Large studies in literature have shown age, sex, T stage, $\mathrm{N}$ stage, grade and perineural invasion as the most common important prognostic variables for parotid gland malignancy (North et al., 1990; Hocwald et al., 2001; Iqbal et al., 2014). In line with the literature data, in our univariate analysis, the OS was prognostic factors with gender, age, $\mathrm{T}$ stage, $\mathrm{N}$ stage, tumor grade, lymphovasculer invasion, perineural invasion, extracapsuler extansion, surgical margin, radiotherapy dose and distant metastasis . The DFS was prognostic factors with gender, $\mathrm{T}$ stage, $\mathrm{N}$ stage, tumor grade, lymphovasculer invasion, perineural invasion, extracapsuler extansion, surgical margin and radiotherapy dose.

In conclusion, We believe that our findings contribute significantly to the awareness of the demographic and pathologic features of parotid gland tumors in the Turkish population and that they are similar to what has been reported elsewhere in the world.

\section{References}

Al-Mamgani A, Van Rooij P, Verduijn GM, et al (2012). Long-term outcomes and quality of life of 186 patients with primary parotid carcinoma treated with surgery and radiotherapy at the daniel den hoed cancer center. Int $J$ Radiat Oncol Biol Phy, 84, 189-95.

Alterio D, Jereczek-Fossa BA, Griseri M, et al (2011). Threedimensional conformal postoperative radiotherapy in patients with parotid tumors: 10 years' experience at the European institute of oncology. Tumori, 97, 328-34.

Feinstein TM, Lai SY, Lenzner D, et al (2011). Prognostic factors in patients with high-risk locally advanced salivary gland cancers treated with surgery and postoperative radiotherapy. Head Neck, 33, 318-23.

Garden AS, El Nagger AK, Morrison WH, et al (1997). Postoperative radiotherapy for malignant tumors of the parotid gland. Int J Radiat Oncol Biol Phys, 37, 79-85.

Hocwald E, Korkmaz H, Yoo GH, et al (2001). Prognostic factors in major salivary gland cancer. Laryngoscope, 111, 1434-9.

Iqbal H, Bhatti AB, Hussain R, et al (2014). Ten year experience with surgery and radiation in the management of malignant major salivary gland tumors. Asian Pac J Cancer Prev, 15, 2195-9.

Ito FA, Ito K, Vargas PA, et al (2005). Salivary gland tumors in a Brazilian population: A retrospective study of 496 cases. Int J Oral Maxillofac Surg, 34, 533-6.

Kaur J, Goyal S, Muzumder S, et al (2014). Outcome of surgery and post-operative radiotherapy for major salivary gland carcinoma: ten year experience from a single institute. Asian Pac J Cancer Prev, 15, 8259-63.

Koivunen P, Suutala L, Schorsch I, et al (2002). Malignant epithelial salivary gland tumors in northern Finland: Incidence 
Mustafa Kandaz et al

and clinical characteristics. Eur Arch Otorhinolaryngol, 259, 146-9.

Lima RA, Tavares MR, Dias FL, et al (2005). Clinical prognostic factors in malignant parotid gland tumours. Otolaryngol Head Neck Surg, 133, 702-708.

Mercante G, Marchese C, Giannarelli D, et al (2014). Oncological outcome and prognostic factors in malignant parotid tumours, J Craniomaxillofac Surg, 42, 59-65.

Nascimento AG, Amaral LP, Prado LA, et al (1986). Mucoepidermoid carcinoma of salivary glands: A clinicopathologic study of 46 cases. Head Neck Surg, 8, 409-17.

North CA, Lee DJ, Piantadosi S, et al (1990). Carcinoma of the major salivary glands treated by surgery or surgery plus postoperative radiotherapy. Int J Radiat OncolBiol Phys, 18, 1319-26.

Terhaard CH, Lubsen H, Rasch CR, et al (2005). Dutch head and neck oncology cooperative group: the role of radiotherapy in the treatment of malignant salivary gland tumors. Int $J$ Radiat Oncol Biol Phys, 61, 103-11. 